\title{
Oblique Incidence of a train of Plane Waves on a Semi-Inflnite Plane.
}

\author{
By H. S. CarsLaw.
}

The problem of the incidence of a train of plane waves on a semi-infinite plane, when the edge of the plane is perpendicular to the direction of the waves, is a two-dimensional one, and was first fully treated in a paper by Sommerfeld.* It was also discussed by me in a paper published in the Proceedings of the London Mathematical Society. $\dagger$

The three-dimensional problem, when the edge of the plane is not perpendicular to the direction of the waves, is a very obvious extension of the method followed in these papers It was suggested to me some years ago by Sommerfeld, and I publish the solution now principally because a long paper + in the current issue of Schlömilch's Zeitschrift für Mathematik und Physik has re-opened the questions of Diffraction which were first solved by this method.

\section{$\$ 1$.}

Let the origin be taken in the edge of the plane, and this line as the axis of $z$, the plane as the plane of $x z$, and the direction from which the waves are coming as the line $(l, m, n)$, or in Spherical Polar Coordinates by the angles $\left(\theta^{\prime}, \phi^{\prime}\right)$.

- Math. Annalen, Bd. xuvrr.-Math. Theorie der Diffraction.

† Proc. Lond. Math. Soc., xxx. - Some Multiform Solutions of Certain Partial Differential Equations.

$¥$ Schlomilch's Zeitschrift, Bd. xLv1.—Über die Beugung der Röntgen. strahlen. A. Sommerfeld. 


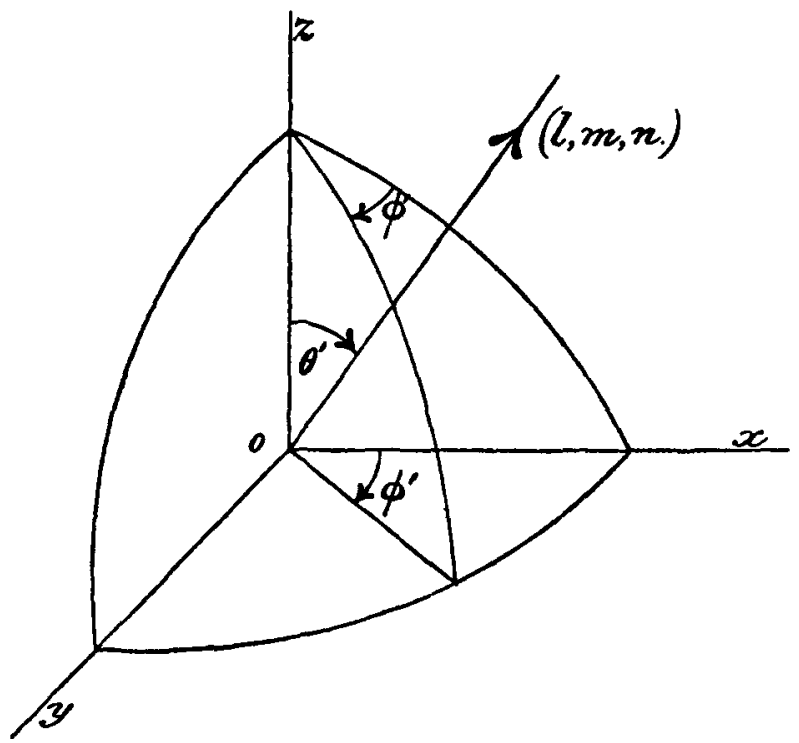

Fig. 1.

Our problem is the investigation of the circumstances which follow when a train of Plane Waves, e.g., Sound Waves, comes in this direction, and strikes upon the plane. We shall find terms in our result corresponding to the waves of Incidence, Reflection, and Diffraction.

The wave-length is supposed finite, so that the approximate methods applied to waves of light are not available.

If the plane were unlimited and not bounded by the straight edge, the problem would present no difficulty. The solution depends upon the equation

$$
\frac{\partial^{2} u}{\partial x^{2}}+\frac{\partial^{2} u}{\partial y^{2}}+\frac{\partial^{2} u}{\partial z^{2}}+\kappa^{2} u=0
$$

$$
\text { with } \frac{\partial u}{\partial y}=0 \text {, at the plane, } y=0 \text {. }
$$

These are satisfied by $u=e^{i x(l x+m y+n z)}+e^{i \kappa(l x-m y+n z)}$.

In the case now considered we proceed from the solution

$$
\begin{aligned}
u_{0} & =e^{i \kappa(l l x+m y+n z)} \\
& =e^{i k r\left(\cos \theta \cos \theta^{\prime}+\sin \theta \sin \theta^{\prime} \cos \left(\phi-\phi^{\prime}\right)\right)}
\end{aligned}
$$

and transform this by the introduction of the complex variable, $a$. 
In this way we obtain, by Cauchy's Theorem,

$$
u_{0}=\frac{1}{2 \pi} \int e^{i \kappa r\left(\cos \theta \cos \theta^{\prime}+\sin \theta \sin \theta^{\prime} \cos (\alpha-\phi)\right)} \frac{e^{i \alpha}}{e^{i \alpha}-e^{i \phi^{\prime}}} d \alpha,
$$

as an equivalent expression for $u_{0}$, the path of integration in the $a$-plane being originally a circuit round the point $a=\phi^{\prime}$, enclosing no other singularity of the integrand.

$$
\text { Thus } u_{0}=\frac{e^{i \kappa r \cos \theta \cos \theta^{\prime}}}{2 \pi} \int e^{i \kappa r \sin \theta \sin \theta^{\prime} \cos (\alpha-\phi)} \frac{e^{i \alpha}}{e^{i \alpha}-e^{i \phi^{\prime}}} d \alpha
$$

Since $\sin \theta$ and $\sin \theta^{\prime}$ are always positive, this integral is of exactly the same form as that ${ }^{*}$ to which we were brought in the discussion of the two-dimensional form. It was then shown that this circuit may be deformed into the path (A) of Fig. 2. It was also shown, and this is the fundamental part of the method, that for this physical problem what we really need is a Multiform Solution, a solution depending on $\phi$ in such a way that the period is $4 \pi$.

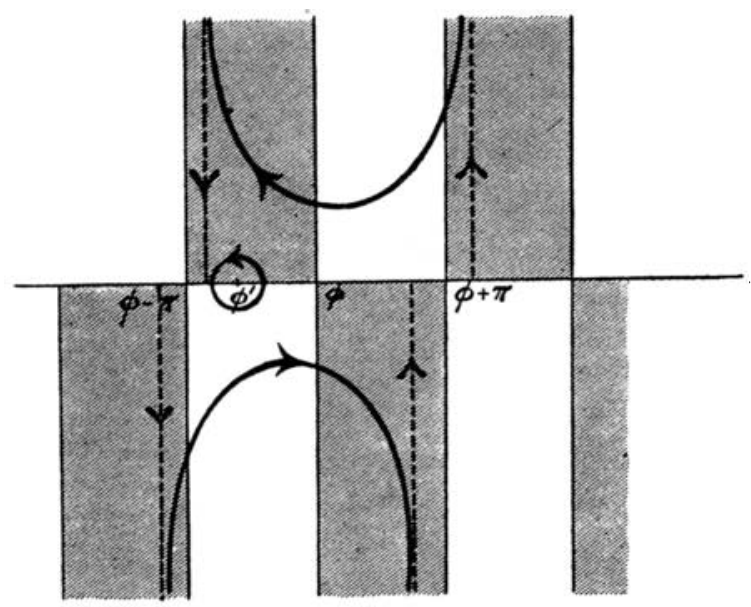

Fig. 2.

Deformation of circuit round $\alpha=\phi^{\prime}$, for $u_{0}$. The small circle round $\phi^{\prime}$ is the original circuit. The path $(A)$ is the two dark curved lines. * $\frac{1}{2 \pi} \int e^{i \kappa r \cos (\alpha-\theta)} \frac{e^{i \alpha}}{e^{i \alpha}-e^{i \theta^{\prime}}} d \alpha . \quad$ See Proc. Lond. Math. Soc., xxx., 
We have such a solution at once in the form

$$
u=\frac{1}{4 \pi} e^{i \kappa r \cos \theta \cos \theta^{\prime}} \int e^{i \kappa r \sin \theta \sin \theta^{\prime} \cos (\alpha-\phi)} \frac{e^{i \alpha}}{e^{\frac{i \alpha}{2}}-e^{i \frac{\phi^{2}}{2}}} d \alpha,
$$

the integral being taken round the path (A), of Fig. 2, corresponding to the value of $\phi$ involved.

(i) This function is a solution of our equation

$$
\nabla^{2} u+\kappa^{2} u=0
$$

since every element of the integrand is a solution; and we have excluded the possibility of infinite values.

(ii) The function is periodic in $\phi$ and of period $4 \pi$ : for when we let $\phi$ change, the path of integration in the $\alpha$-plane is only pushed parallel to itself in the direction of the real axis of $a$ : and when $\phi$ is increased by $4 \pi$, the integrand itself returns to its original value.

(iii) The function is finite and continuous for all values of $r$.

This follows from the choice of our path (A).

(iv) When $\left|\phi-\phi^{\prime}\right|<\pi$, the function takes the value $u_{0}$ at $r=\infty$; when $\pi<\left|\phi-\phi^{\prime}\right|<3 \pi$, the value at $r=\infty$ is zero.

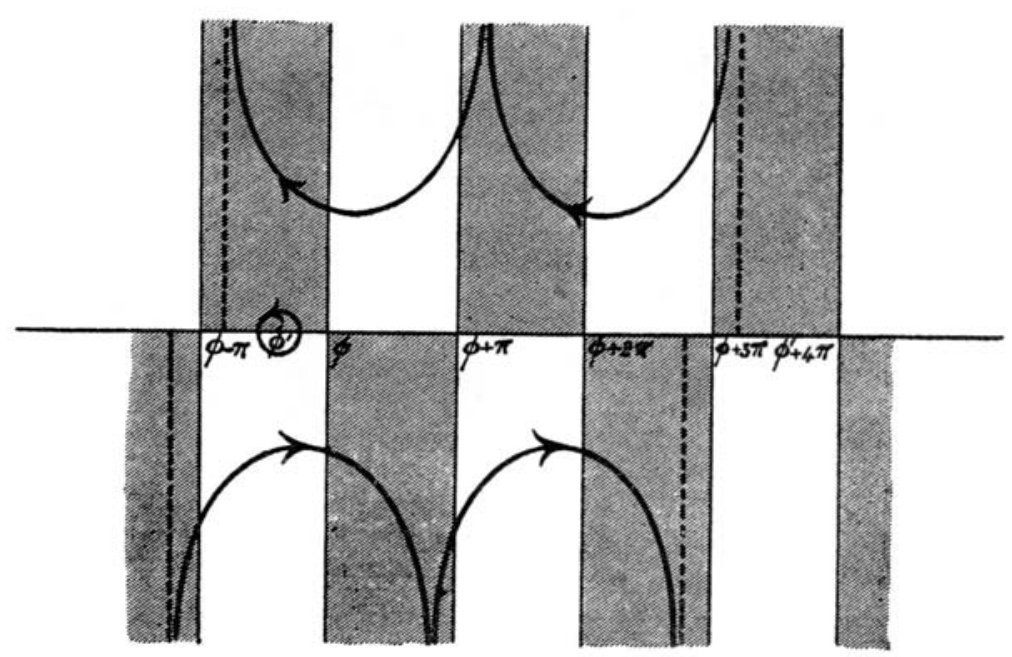

Fic. 3.

The dark curved lines denote the path (A) for the values $\phi$ and $\phi+2 \pi$ when the period is $4 \pi$. 
The proof of this property follows from Fig. 3 . In the first case the path (A) of integration is deformable into the two rectilineal parts, in which the real part of the exponential is negative, and thus vanishes when $r=\infty$; and we have in addition the circuit round the singular point $a=\phi^{\prime}$, this circuit giving the original value $u_{0}$. In the second case there is no singular point within the closed circuit with which we have completed the path (A); and, hence, when $r=\infty$ that integral now vanishes.

When we associate with this value of $u$, which we may call $u\left(\phi^{\prime}\right)$, that which corresponds to the value $-\phi^{\prime}$, say $u\left(-\phi^{\prime}\right)$, we obtain

$$
u=u\left(\phi^{\prime}\right)+u\left(-\phi^{\prime}\right) \text {, }
$$

a solution of our equation $\nabla^{2} u+\kappa^{2} u=0$, with the condition

$$
\frac{\partial u}{\partial \phi}=0 \text { at } \phi=0 \text { and } \phi=2 \pi,
$$

and we are enabled to write down the velocity potential $\Phi$ of our physical problem.

$$
\$ 2 .
$$

The integral corresponding to (3) was fully discussed in Sommerfeld's original paper.* The space with which we are concerned, namely.

$$
\left.\begin{array}{l}
0<r<\infty \\
0<\theta<\pi \\
0<\phi<2 \pi
\end{array}\right\}
$$

bas to be considered in three parts-

$$
\begin{aligned}
& \text { (A) } 0<\phi<\pi-\phi^{\prime} \text {, } \\
& \text { (B) } \pi-\phi^{\prime}<\phi<\pi+\phi^{\prime}, \\
& \text { (C) } \pi+\phi^{\prime}<\phi<\quad 2 \pi .
\end{aligned}
$$

* Wath. Ann., Bd. Xlvir., pp. 357-374. 
In (A) we find

$$
\begin{aligned}
\Phi= & \cos 2 \pi\left[\frac{r}{\lambda}\left(\cos \theta \cos \theta^{\prime}+\sin \theta \sin \theta^{\prime} \cos \left(\phi-\phi^{\prime}\right)\right)+\frac{t}{\tau}\right] \\
& +\cos 2 \pi\left[\frac{r}{\lambda}\left(\cos \theta \cos \theta^{\prime}+\sin \theta \sin \theta^{\prime} \cos \left(\phi+\phi^{\prime}\right)\right)+\frac{t}{\tau}\right] \\
& -\frac{1}{4 \pi} \sqrt{\frac{\lambda}{r \sin \theta} \sin \theta^{\prime}}\left(\frac{1}{\cos \left(\frac{\phi-\phi^{\prime}}{2}\right)}+\frac{1}{\cos \left(\frac{\phi+\phi^{\prime}}{2}\right)}\right) \cos \left(2 \pi\left(\frac{r}{\lambda} \cos \left(\theta+\theta^{\prime}\right)+\frac{t}{\tau}\right)-\frac{\pi}{4}\right) .
\end{aligned}
$$

In (B) we find

$$
\begin{aligned}
\Phi= & \cos 2 \pi\left[\frac{r}{\lambda}\left(\cos \theta \cos \theta^{\prime}+\sin \theta \sin \theta^{\prime} \cos \left(\phi-\phi^{\prime}\right)\right)+\frac{t}{\tau}\right] \\
& -\frac{1}{4 \pi} \sqrt{\frac{\lambda}{r \sin \theta} \sin \theta}\left(\frac{1}{\cos \left(\frac{\phi-\phi^{\prime}}{2}\right)}+\frac{1}{\cos \left(\frac{\phi+\phi^{\prime}}{2}\right)}\right) \cos \left(2 \pi\left(\frac{r}{\lambda} \cos \left(\theta+\theta^{\prime}\right)+\frac{t}{\tau}\right)-\frac{\pi}{4}\right) .
\end{aligned}
$$

In (C) we tind

$\Phi=-\frac{1}{4 \pi} \sqrt{\frac{\lambda}{r \sin \theta \sin \theta^{\prime}}}\left(\frac{1}{\cos \left(\frac{\phi-\phi^{\prime}}{2}\right)}+\frac{1}{\cos \left(\frac{\phi+\phi^{\prime}}{2}\right)}\right) \cos \left(2 \pi\left(\frac{r}{\lambda} \cos \left(\theta+\theta^{\prime}\right)+\frac{t}{\tau}\right)-\frac{\pi}{4}\right)$

where $\lambda$ and $\tau$ are the wave-length and period of the incident-wave. Hence we may regard the disturbance in the region (A) as made up of three parts

(i) The Incident Wave-in the direction $(l, m, n)$, or $\left(\theta^{\prime}, \phi^{\prime}\right)$;

(ii) The Reflected Wave-in the direction $\left(\theta^{\prime},-\phi^{\prime}\right)$;

(iii) A Series of Waves proceeding outwards from the edge of the Plane.

In (B) the Reflected Wave does not enter, and in (C) we have only what we may call the Diffracted Wave.

No records of experimental investigations of this physical problem are to be had. It would be interesting to see to what extent they would agree with our results, as in the two-dimensional case the polarising effects of the Diffraction are fully recognised in the mathematical formulae. The only observations which at all 
bear upon it are those of Maey, ${ }^{*}$ who deals with rays proceeding from a point, not plane waves such as we have had to consider. He finds that the edge of the screen appears brighter when the direction of incidence and diffraction are supplementary. This may appear to be illustrated in our problem by the fact that when $\theta+\theta^{\prime}=\pi$, the diffracted waves take the simple form of a plane wave proceeding from the origin whose amplitude changes with the distance from the origin.

* Wied. Ann., Bd. XLIX., p. 91.-Über die Beugung des Lichtes, an einem geraden scharfen Schirmrande. 